\title{
Isolated Pituitary Stalk Relapse of Primary Penile Lymphoma
}

\author{
Yasuhiro Tanaka, Atsushi Tanaka, Akiko Hashimoto and Isaku Shinzato
}

\begin{abstract}
A 76-year-old Japanese man was admitted to our hospital because of the occurrence of multiple nodules on the shaft of his penis. He was diagnosed with diffuse large B cell lymphoma (DLBCL). His lymphoma was located only in his penis. He received immunochemotherapy and involved-field radiotherapy, and achieved complete response (CR). About two years later, he complained of a poor appetite. Magnetic resonance imaging showed a mass lesion in the pituitary stalk. Biopsy of this mass revealed the recurrence of DLBCL. He received whole-brain radiotherapy, and achieved CR. This is the first case of an isolated pituitary stalk relapse of primary penile lymphoma.
\end{abstract}

Key words: primary penile lymphoma, diffuse large B cell lymphoma, central nervous system relapse, pituitary stalk

(Intern Med 56: 835-839, 2017)

(DOI: 10.2169/internalmedicine.56.7788)

\begin{abstract}
Introduction
Primary penile lymphoma (PPL) is extremely rare, and about fifty cases have been reported thus far (1). This type of lymphoma commonly localizes in the penis. In almost all cases, the histological type was diffuse large B cell lymphoma (DLBCL). The treatment modality is mainly chemotherapy and radiotherapy. In some cases, it relapses in regions other than the penis, but rarely in the central nervous system (CNS).

We herein report a case of isolated pituitary stalk relapse of PPL. The localized penile lymphoma was successfully treated by rituximab combination chemotherapy and involved-field radiotherapy, and the patient achieved complete response. However, about two years later, the lymphoma relapsed as a mass lesion in the pituitary stalk. Open biopsy of the mass lesion confirmed the diagnosis of lymphoma relapse. To our knowledge, this is the third case of isolated relapse in CNS and the first case of an isolated pituitary stalk relapse of PPL.
\end{abstract}

Case Report
A 76-year-old man was admitted to our hospital because
of pain and swelling of his penis in February 2013. He had
type II diabetes mellitus treated by subcutaneous insulin in-
jection. He noticed swelling of the glans penis and multiple
nodules on the shaft of his penis in December 2012. Physi-
cal examinations revealed swelling of the foreskin and glans
penis with marked ulceration of the glans penis and multiple
nodules on the shaft of his penis. He had no superficial lym-
phadenopathy or abnormal findings in his thoracic or ab-
dominal cavities. Laboratory tests showed elevated levels of
only C-reactive protein (CRP, 0.8 mg/dL) and hemoglobin
A1c (HbA1c, 8.3\%); those of lactate dehydrogenase (LDH)
and soluble interleukin-2 receptor (sIL-2R) were within the
normal range. Magnetic resonance imaging (MRI) revealed a
soft-tissue mass in the dorsal area of the glans penis and
shaft. This mass showed mild heterogeneous high intensity
on short tau inversion recovery (STIR) T1-weighted images
(Fig. 1a), mild heterogeneous low intensity on T2-weighted
images, and high intensity on diffusion-weighted images.
The remarkable swelling of his foreskin was also present
(Fig. 1b). Biopsy of the nodules on the shaft of his penis

Department of Hematology and Clinical Immunology, Nishi-Kobe Medical Center, Japan

Received for publication May 31, 2016; Accepted for publication July 24, 2016

Correspondence to Dr. Yasuhiro Tanaka, ytanaka77@nmc-kobe.org 
(a)

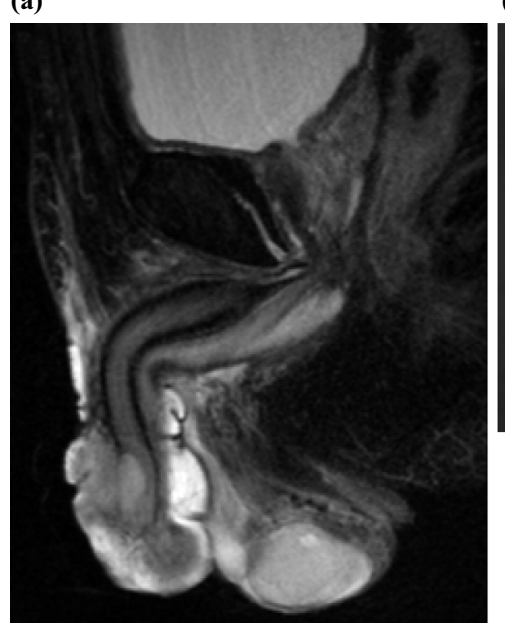

(b)

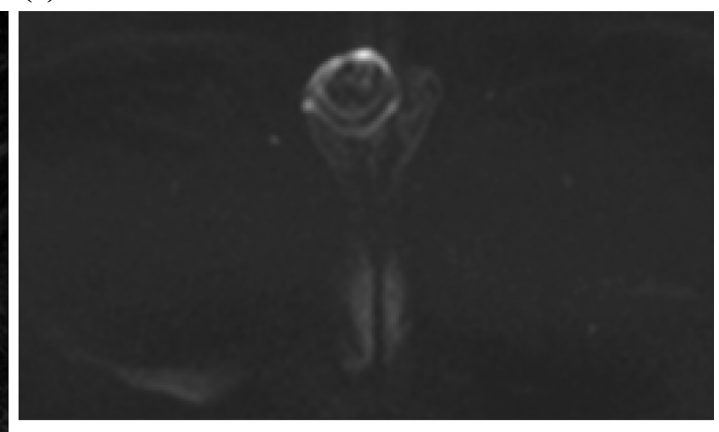

Figure 1. MR images of the penis before treatment. (a) A sagittal section of a STIR T1-weighted image shows a soft tissue mass with mild heterogeneous high intensity in the dorsal area of the glans penis and shaft. (b) A coronal section of a diffusion-weighted image shows high-intensity swelling of the foreskin.

(a)

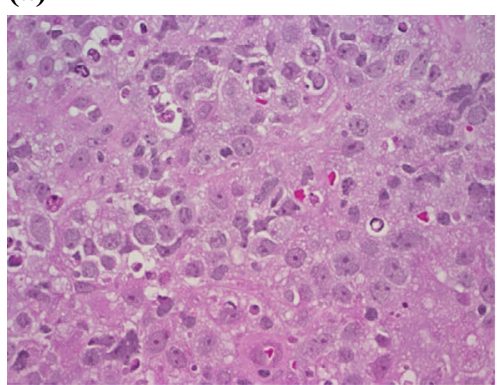

(d)

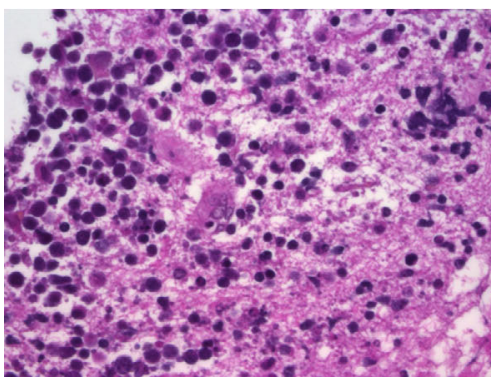

(b)

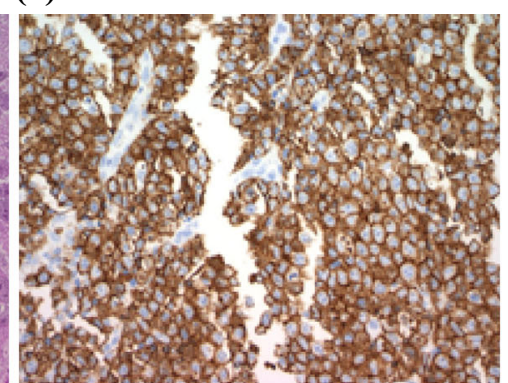

(e)

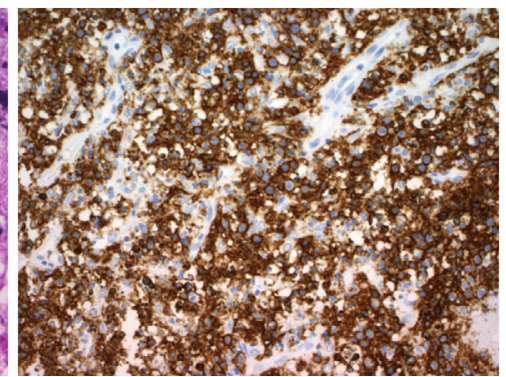

(c)

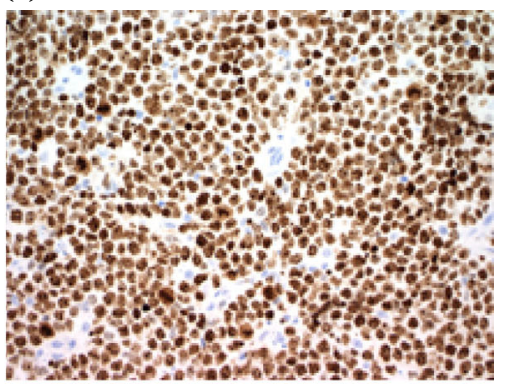

(f)

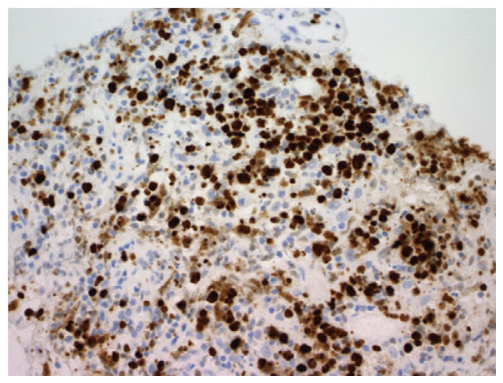

Figure 2. The histopathological findings of the biopsied sample obtained from a nodule in the shaft and pituitary stalk. (a) - (c) are pathological pictures of the nodule in the shaft. (d) - (f) are pathological pictures of the mass in the pituitary stalk. (a) Numerous abnormal cells with scant cytoplasm and clear nucleoli were densely occupied with a scattered starry sky-like appearance in the nodule [Hematoxylin and Eosin (H\&E) staining, original magnification of objective lens, 40× ]. (b, e) In immunohistochemical staining, CD20 was positive in the abnormal cells (original magnification of objective lens, 20x). (c, f) The Ki-67 index was very high (original magnification of objective lens, 20x). (d) The numerous abnormal cells seen in the nodule of the shaft densely infiltrated the pituitary stalk (H\&E staining, original magnification of objective lens, 40x).

showed that numerous abnormal cells with scant cytoplasm and clear nucleoli were densely occupied with a scattered starry sky-like appearance mainly involving the subcutaneous tissue and dermis (Fig. 2a). The abnormal cells were exclusively found outside of the small vessels in the biopsied subcutaneous tissue. An immunohistochemical analysis revealed that these abnormal cells were positive for CD20 (Fig. 2b), CD10, and BCL6, and negative for CD5 and BCL2, which confirmed the diagnosis of DLBCL, germinal center B cell type according to Hans' criteria (2). The Ki-67 
(a)

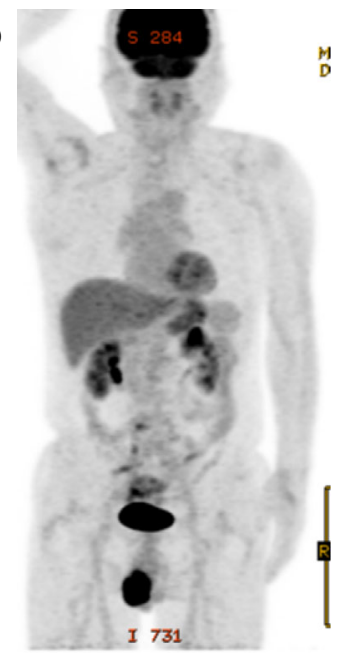

(b) $=2$

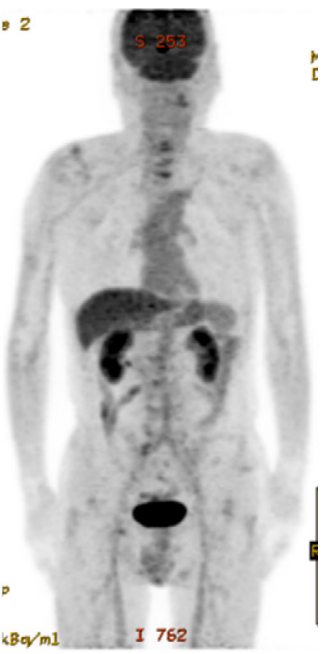

Figure 3. Whole-body PET-CT scans. (a) Abnormal accumulation of FDG in the penis was only found before the treatment. (b) No abnormal accumulation of FDG was found in the whole body at the time of the pituitary stalk relapse.

index was very high (Fig. 2c). A fluorescence in situ hybridization (FISH) analysis revealed no MYC split signals in these lymphoma cells. Positron emission tomographycomputed tomography (PET-CT) showed marked accumulation of fluorodeoxyglucose (FDG) only in his penis (Fig. 3a). A bone marrow examination did not show any abnormal cells. He was diagnosed with PPL at clinical stage I EA. The international prognostic index (IPI) (3) was classified as low risk.

Therapy with rituximab combination THP-COP (R-THPCOP, cyclophosphamide, $650 \mathrm{mg} / \mathrm{m}^{2}$; pirarubicin, $40 \mathrm{mg} / \mathrm{m}^{2}$; vincristine, $1 \mathrm{mg} / \mathrm{m}^{2}$, and oral prednisolone, $1 \mathrm{mg} / \mathrm{kg}$ every 5 days) was started in March 2013. After three courses of RTHP-COP therapy, MRI of the penis showed shrinkage of the soft-tissue mass, suggesting a partial response (PR). Involved-field radiotherapy (IFRT; total, 40 Gy over 20 fractions) was applied after the immunochemotherapy. MRI of the penis revealed no abnormal intensity signals in his penis, and PET-CT revealed no abnormal accumulation of FDG. He achieved complete response (CR) in October 2013.

About two years after achieving CR, he was readmitted to our hospital because of appetite loss and body weight loss of $10 \mathrm{~kg}$ over the past month in January 2016. PET-CT revealed no abnormal uptake of FDG in the whole body including his penis (Fig. 3b). MRI of the brain showed an abnormal mass lesion in the pituitary stalk. This mass showed mild homogeneous low intensity on T1-weighted images and mild homogeneous high intensity on T2-weighted images. The mass lesion was homogeneously gadoliniumenhanced (Fig. 4a). No abnormal cells were found in the cerebrospinal fluid. The basal levels of thyroid-stimulating hormone (TSH $0.024 \mu \mathrm{IU} / \mathrm{mL}$, normal range 0.390-4.01), adrenocorticotropic hormone (ACTH $3.8 \mathrm{pg} / \mathrm{mL}$, normal range 7.2-63.3), and cortisol $(0.6 \mu \mathrm{g} / \mathrm{dL}$, normal range 4.019.3) were low; and the basal level of prolactin (34.39 ng/

$\mathrm{mL}$, normal range 3.58-12.78) was high. The basal levels of growth hormone $(\mathrm{GH} 1.56 \mathrm{ng} / \mathrm{mL}$, normal range below 2.47), luteinizing hormone ( $\mathrm{LH} 0.21 \mathrm{mIU} / \mathrm{mL}$, normal range 0.79-5.72), and follicle-stimulating hormone (FSH 0.92 $\mathrm{mIU} / \mathrm{mL}$, normal range 2.00-8.30) were within the normal range. The loading test with $100 \mu \mathrm{g}$ of growth hormonereleasing hormone $(\mathrm{GRH}), 200 \mu \mathrm{g}$ of thyrotropin-releasing hormone (TRH), $100 \mu \mathrm{g}$ of luteinizing hormone-releasing hormone (LH-RH), and $100 \mu \mathrm{g}$ of corticotropin-releasing hormone (CRH) revealed rapid responses of GH, LH, FSH, and prolactin, but no response of TSH, a result consistent with hypopituitarism. We did not examine the vasopressin secretion because he did not demonstrate either polyuria or polydipsia.

Open biopsy of the mass lesion confirmed the diagnosis of DLBCL with the same immunohistochemical phenotype as the initial nodules of his penis (Fig. 2d-f). After open biopsy, he received hormone replacement therapy using thyroxine and dexamethasone instead of hydrocortisone for the treatment of postoperative brain edema. We chose radiotherapy instead of chemotherapy because his performance status (PS) was low, and he became bed-ridden after open biopsy. He received whole-brain radiotherapy, and MRI of the pituitary showed the disappearance of the mass (Fig. 4b). However, he developed methicillin-resistant Staphylococcus aureus (MRSA) sepsis concomitant with purulent spondylitis and epidural abscess at the $\mathrm{C} 5$ level and received intravenous administration of vancomycin for about three months. At the time of writing, he is alive and has maintained CR for his lymphoma for about three months since open biopsy, but he remains bed-ridden due to left hemiparesis as sequelae of the epidural abscess.

\section{Discussion}

We herein report a case of isolated pituitary stalk relapse of primary penile DLBCL.

Non-Hodgkin's lymphomas (NHLs) typically originates from lymph nodes, but extranodal NHL may involve many organs, such as the gastrointestinal tract, thyroid, and CNS (4). In particular, involvement of the penis is rare among extranodal NHLs. To date, almost 50 cases have been reported in the medical literature, and Chu et al. summarized the characteristics of PPL from the literature (1). The most common histopathological type of PPL was DLBCL. The shaft of the penis and the glans penis were most commonly involved. The most common presentation was a mass or nodules in the penis. PPL in almost all reported cases was localized in the penis at clinical stage I EA. The treatment modality was mainly chemotherapy or radiotherapy. The prognosis was relative good after achieving CR. However, relapse of PPL exclusively in the CNS is rare.

Only two cases of isolated CNS relapse of PPL have been reported, although another case of PPL involving the brain and lung at the initial diagnosis was reported (5). Chu et 
(a)

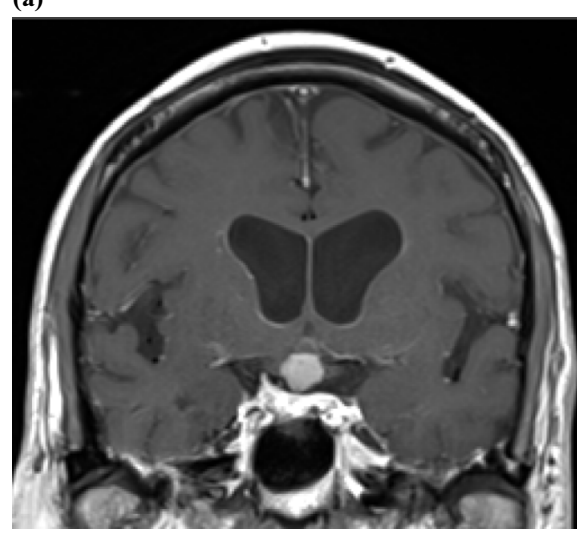

(b)

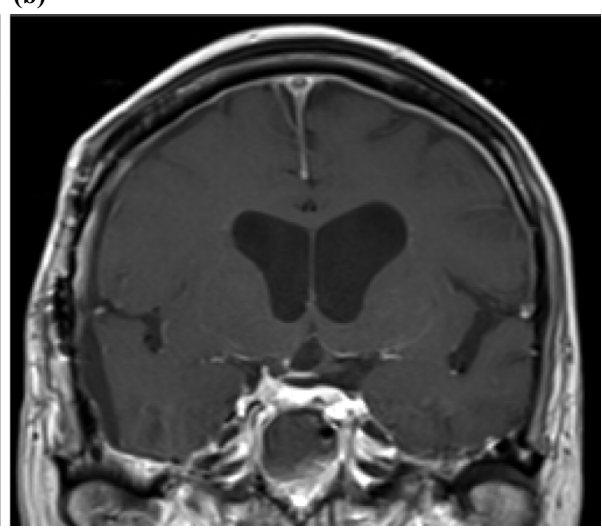

Figure 4. Coronal sections of pituitary MR images. (a) The mass in pituitary stalk was homogeneously gadolinium-enhanced before open biopsy. (b) The mass in the pituitary stalk disappeared after whole-brain radiotherapy. Subdural liquid collection was observed around the right temporal lobe after open biopsy.

al. (1) reported the case of a 72-year-old man diagnosed with primary penile DLBCL at stage I E. Four cycles of systemic chemotherapy were administered, and he achieved CR. About two years later, he developed a fever with an elevated level of $\mathrm{LDH}$ and presented with transient obnubilation, left limb hypodynamia, mild headache, nausea, and vomiting. MRI showed many space-occupying lesions in the bilateral hemispheres of the brain. He died of refractory lymphoma. Beal and Mears (6) reported another case of a 64-year-old man with primary penile DLBCL at stage I E about six months after local injection for erectile dysfunction. Three cycles of systemic chemotherapy and fractionated radiotherapy were administered, and he achieved CR. One year later, his lymphoma relapsed in the shaft of his penis, which was outside of the previously treated area. He received five courses of systemic chemotherapy and fractionated radiotherapy, and he achieved CR again. However, he was found to have diffuse cerebral involvement after one course of rituximab. He is alive with stably controlled cerebral disease. Generally, DLBCL very commonly relapses in the CNS, particularly in the parenchymal brain $(7,8)$. Unlike the previous case, our case had relapse in the pituitary stalk. The pituitary stalk is very rarely involved in cases of relapse of DLBCL, although the pituitary stalk is occasionally involved in primary lymphoma of the pituitary (9). To our knowledge, no other case of isolated pituitary stalk relapse of DLBCL outside of the CNS has been reported. Therefore, this is a very unusual phenomenon of relapse in DLBCL.

Several groups have reported the risk factors for CNS relapse of NHL. The representative factors are age, more than two extranodal sites, and LDH level elevation, which consisted of IPI $(8,10)$. In our patient, the IPI was low, and no risk factors for CNS relapse except for age were found. However, the initial involved site of NHL is another risk factor for CNS relapse. The testis was the established site for CNS relapse, despite the low IPI and early stage at the initial diagnosis (11). The urogenital track was the sanctuary of anti-cancer drugs because of the low penetration of the drugs into tissues. It has been reported that NHL arising from other sites in the urogenital track except the testis can relapse in the CNS, but its frequency is very low. In the case of primary vaginal lymphoma, Hayase et al. reported only one case of isolated relapse in the CNS in 2011 (12). Boehme et al. reported that 2 out of 17 primary bladder lymphomas, 3 out of 17 primary adrenal lymphomas, and 3 out of 26 primary renal lymphomas relapsed in the CNS (13). Therefore, although rarely reported, NHL arising from the urogenital track can relapse in the CNS.

Why NHL arising from the urogenital track, including the penis, can relapse in the CNS is unknown. Physicians should be aware of the very rare pattern of relapse in the CNS for NHL arising from the urogenital track, including the penis, even after achieving CR. Collections of cases of NHL arising from the urogenital track relapsing in the CNS may clarify the necessity of prophylaxis against relapse in the CNS at the initial diagnosis in the future.

The authors state that they have no Conflict of Interest (COI).

\section{Acknowledgement}

We thank Dr. Masamitsu Nishihara for performing the open biopsy of the pituitary stalk.

\section{References}

1. Chu L, Mao W, Curran Vikramsingh K, et al. Primary malignant lymphoma of the glans penis: a rare case report and review of the literature. Asian J Androl 15: 571-572, 2013.

2. Hans CP, Weisenburger DD, Greiner TC, et al. Confirmation of the molecular classification of diffuse large B-cell lymphoma by immunohistochemistry using a tissue microarray. Blood 103: 275282, 2004.

3. The International Non-Hodgkin's Lymphoma Prognostic Factors Project. A predictive model for aggressive non-Hodgkin's lymphoma. N Engl J Med 329: 987-994, 1993. 
4. Stein H. Diffuse large B-cell lymphoma (DLBCL), NOS. In: WHO Classification of Tumors of Haematopoietic and Lymphoid Tissues. 4th ed. Swerdlow SH, Campo E, Harris NL, et al., Eds. Lyon, IARC Press, 2008: 233-237.

5. Gong Z, Zhang Y, Chu H, et al. Priapism as the initial symptom of primary penile lymphoma: a case report. Oncol Lett 8: 19291932, 2014

6. Beal K, Mears JG. Short report: penile lymphoma following local injections for erectile dysfunction. Leuk Lymphoma 42: 247-249, 2001.

7. Doolittle ND, Abrey LE, Shenkier TN, et al. Brain parenchyma involvement as isolated central nervous system relapse of systemic non-Hodgkin lymphoma: an International Primary CNS Lymphoma Collaboration Group report. Blood 111: 1085-1093, 2008.

8. Feugier P, Virion JM, Tilly $\mathrm{H}$, et al. Incidence and risk factors for central nervous system occurrence in elderly patients with diffuse large-B-cell lymphoma: influence of rituximab. Ann Oncol 15: 129-133, 2004

9. Giustina A, Gola M, Doga M, Rosei EA. Clinical review 136: Primary lymphoma of the pituitary; an emerging clinical entity. J Clin Endocrinol Metab 86: 4567-4575, 2001.

10. Shimazu Y, Notohara K, Ueda Y. Diffuse large B-cell lymphoma with central nervous system relapse: prognosis and risk factors according to retrospective analysis from a single-center experience. Int J Hematol 89: 577-583, 2009.

11. Zucca E, Conconi A, Mughal TI, et al. Patterns of outcome and prognostic factors in primary large-cell lymphoma of the testis in a survey by the International Extranodal Lymphoma Study Group. J Clin Oncol 21: 20-27, 2003.

12. Hayase E, Kurosawa M, Yonezumi M, Suzuki S. Early relapse in the central nervous system-after achieving complete response in primary vaginal lymphoma. Rinsho Ketsueki 53: 229-234, 2012 (in Japanese, Abstract in English).

13. Boehme V, Zeynalova $\mathrm{S}$, Kloess $\mathrm{M}$, et al. Incidence and risk factors of central nervous system recurrence in aggressive lymphoma: a survey of 1693 patients treated in protocols of the German HighGrade Non-Hodgkin's Lymphoma Study Group (DSHNHL). Ann Oncol 18: 149-157, 2007.

The Internal Medicine is an Open Access article distributed under the Creative Commons Attribution-NonCommercial-NoDerivatives 4.0 International License. To view the details of this license, please visit (https://creativecommons.org/licenses/ by-nc-nd/4.0/).

(C) 2017 The Japanese Society of Internal Medicine http://www.naika.or.jp/imonline/index.html 